\title{
A practical cost-based approach to determining reimbursement rates: a necessary component of effective payment reform
}

This article was published in the following Dove Press journal:

Innovation and Entrepreneurship in Health

19 January 2015

Number of times this article has been viewed

\author{
Keith A Joiner ${ }^{1}$ \\ Theresa R Kramer ${ }^{2}$ \\ Robin Cooper ${ }^{3}$ \\ 'College of Medicine and Eller \\ College of Management, The \\ University of Arizona, Tucson, AZ, \\ USA; ${ }^{2} O$ phthalmology Resident \\ Clinic, Krieger Eye Institute, Life \\ bridge Health, Baltimore, MD, USA; \\ ${ }^{3}$ Nottingham University Business \\ School, The University of Nottingham, \\ Nottingham, UK
}

\begin{abstract}
Payment reform is intended to decrease national health care spending by limiting costs at the provider level, while maintaining quality. The underlying cost basis for current payment reform initiatives is revenue, charges or claims, all of which are proxies for cost, not measured cost. Methods for costing of health care services and procedures are established, not standardized. We propose the creation of an internet-cloud-based, national level, open-source health care cost system for data mining of reported costs of health care services and procedures. This data set can be applied to any payment reform initiative, and will inform policy decisions on health care spending.
\end{abstract}

Keywords: reported costs, payment reform, activity-based costing

\section{Current concept of cost in health care}

Payment reform is a central component of health care reform. A basic premise of payment reform is that current trajectories for increases in spending are unsustainable. These trajectories are in large part due to current payment mechanisms. ${ }^{1,2}$ Most experts agree $^{3}$ that it will not be possible, in the current fee for service payment system, to bend the cost curve, while maintaining or improving quality. An array of new models, such as bundled/episode of care payments, ${ }^{4}$ defined contribution and reference pricing plans ${ }^{5,6}$ shared savings plans, and more are intended to limit expenditures, by setting spending targets and/or payment levels to predetermined amounts. Simultaneously, these new payment models are influencing the organization of the delivery systems, driving consolidation and integration of providers, hospitals, and payers. Arguably the central issue in these transitions is how to set spending targets/payment levels to simultaneously facilitate quality and efficiency without shifting unacceptable levels of risk to providers, curtailing needed care, or reducing salaries and/or profit margins below sustainable levels. ${ }^{7}$ Hence, the methodology for setting and adjusting payment levels is central to the acceptability to consumers, providers, and payers in partially or fully capitated payment models, and as components of accountable and integrated care.

Payment reform is intended to improve the value of health care spending. "Since value is defined as outcomes relative to costs", 8 it encompasses efficiency. Health care spending in the US is considered both productively and allocatively inefficient. As summarized by Baicker and Chandra:

Productive efficiency (means) that health care resources are put to the best use possible and produce as much health as they can, and allocative efficiency (means) that the right amount of resources are being devoted to health care, versus other goods in the economy.
McClelland Hall, Room 4II II 30 Helen

St, Tucson, Arizona, USA 85721

Tel +5206264655

Email kjoiner@email.arizona.edu 
[...] It is not possible to achieve allocative efficiency without productive efficiency [...] It is impossible to evaluate allocative inefficiency in the presence of productive inefficiency. ${ }^{9}$

This argument, along with many others, suggests that the focus on cost control should be on preferentially enhancing productive efficiency, rather than on the more nuanced issue of determining the right amount to spend (ie, the fraction of GDP Gross Domestic product represented by health care spending). In fact, it is estimated that up to one third of all health care spending in the US is productively inefficient. ${ }^{10}$ Resources are being devoted to activities that are unnecessary, at best, or harmful, at worst - they are not being put to the best use possible or producing as much health as they can.

The fundamental challenge is to discriminate valuable from wasteful spending. Doing so is fraught with controversy in any health care system, but is particularly difficult in the US. Multiple broad-based initiatives, including Choosing Wisely, ${ }^{11}$ Clinical Evidence, ${ }^{12}$ Less is More, ${ }^{13}$ National Institate for Health and Care Excellance, ${ }^{14}$ and recent reviews, ${ }^{15}$ focus on identifying specific procedures that provide minimal or no benefit. Voluntary adoption of the recommendations by physicians, based on guidance from these sources, is one approach, but it suffers from slow adoption, and lack of generalizability across the gamut of resource utilization in health care.

Effectively all current calculations on the efficiency (or inefficiency) or value of spending use cost estimates derived from revenues. What is rarely considered, or at least discussed, is whether the attribution methodology for calculating costs is accurate and/or appropriate. The different perspectives on the value of health care spending over decades take it as a given that revenues are an accurate reflection of costs. Even with payment reform initiatives, payment levels and cost targets are typically set based on current payment models - in particular, fee for service medicine. For example, the shared savings model for accountable care organizations sets cost targets by determining Medicare spending (revenues) in the relevant geographic area over the preceding 3 years. The Prometheus payment model, ${ }^{4}$ which allocates payments for episodes of care, derives payments from charges. (Of note, the most substantial impediments to implementation of the Prometheus model are missing or inaccurate claims data, ${ }^{16}$ and/or wide variations across sites). ${ }^{17}$ Penalties and incentives for hospital readmission rates are scaled to current hospital Medicare reimbursements. Medicare Advantage payment reform includes adjusting Medicare Advantage payments to Part A and B Medicare revenues. Patient centered medical home payment models typically provide a "management fee" for services not typically covered by claims. While the fee may be derived from estimating the costs of additional infrastructure required to fulfill Patient Centered Medical Home PCMH stipulations, the central payment system is fee for service or at least predicated on revenues. Value-based purchasing builds in financial penalties or incentives for quality, using revenues to set both the baseline and the incremental changes. Transparent pricing ${ }^{2}$ encourages ready availability of negotiated prices, but those are typically determined based on some estimation of revenues. To summarize, all current major payment reform/cost control initiatives are predicated on using revenues as a proxy for costs.

Instituting payment reform and setting cost targets based on revenues implicitly assumes this is an effective approach for projecting actual costs. TK, RC Two of us ${ }^{18,19}$ have previously pointed out the problems and consequences of using such revenue-based cost assignment ${ }^{19}$ in clinical practice. $\mathrm{RBCA}$ is based on the invalid assumption that all procedures earn the same profit margin, and in so doing induces a systematic bias in the way indirect costs are assigned. For example, assume that a medical facility performs three different procedures (A, B, and $\mathrm{C}$ ) (Table 1). The total reimbursement for the period is US $\$ 1,000$. Procedure A was responsible for $\$ 200$, procedure B for $\$ 300$, and procedure $C$ for $\$ 500$. If the total cost of all three procedures was $\$ 400$, then the overall profit was $\$ 600$ giving an average profit margin of $60 \%$. The cost assignment process is straightforward; the $\$ 400$ of total cost is divided by the total revenue of $\$ 1,000$ to give dollars of cost per dollar of reimbursement of 0.40 . The reported costs (rcosts) of the three procedures are $\$ 80, \$ 120$, and $\$ 200$ respectively and their respective profits are $\$ 120, \$ 180$, and $\$ 300$. The profit margin for all three procedures is the same

Table I Calculation of reported procedure costs and profit margins using Revenue Based Cost Assignment

\begin{tabular}{llllll}
\hline Procedure & Reimbursements (USD) & Cost per dollar revenue & Reported cost & Profit & Profit margin \\
\hline A & $\$ 200$ & 0.40 & $\$ 80$ & $\$ 120$ & $60 \%$ \\
B & $\$ 300$ & 0.40 & $\$ 120$ & $\$ 180$ & $60 \%$ \\
C & $\$ 500$ & 0.40 & $\$ 200$ & $\$ 300$ & $60 \%$ \\
Total & $\$ 1,000$ & & $\$ 400$ & $\$ 600$ & $60 \%$ \\
\hline
\end{tabular}


at $60 \%$, which is the average profit margin for the facility. In reality, the profit margins for the three procedures can be distributed using any methodology, as long as they average to $60 \%$. Our proposal outlines an approach to align profit margin with costs, rather than reimbursements.

Financial incentives or profits are misaligned ${ }^{20,21}$ leading to the rise of specialty and procedure based medicine and decline in the attractiveness of primary care. This has profound implications for providers, in moving from a fee for service system, toward more global payment mechanisms. In fee for service medicine, the intrinsic incentive is to do more. Increasing utilization of a higher profit margin, more specialized interventions can cross-subsidize shortfalls from lower profit margin activities, without regard to costs. With global payment mechanisms, the intrinsic incentive is to do less, and to minimize utilization of more specialized interventions. As the payment models change, determining the optimal mix of services to balance quality and profitability will be difficult if not impossible without knowing actual costs.

This conundrum is equally applicable to consumers. Consumer-directed health care ${ }^{22}$ is intended to encourage consumers to put more "skin in the game", by asking them to make decisions on opportunity costs for health and health care related expenditures. This philosophy is implemented by increasing consumer cost-sharing, through high deductible health care plans, increased co-pays and co-insurance and more. Partially as a consequence, discretionary spending has decreased, for both needed and unneeded services. While value based insurance design is intended to differentially set consumer cost-sharing requirements based on the expected value of the service, pharmaceutical or intervention, costsharing is still set relative to expenditures, not measured cost. If consumers are being asked to take on more costs should these not be actual or appropriately measured costs?

\section{How did we get here?}

The reasons cost has never been measured in health care are numerous. Arguably the most important is the historical development of Medicare, ${ }^{23}$ and insurance acting as a buffer between cost and pricing. The Medicare payment system was initially based on usual and customary charges or "what the provider said they needed to provide the service". It became an unregulated but massively subsidized industry where charges were entirely at the discretion of the provider.

Attempts to modulate the unregulated rise in national expenditure on health care were based on the introduction of the relative value unit. ${ }^{24}$ At that time there were no highly accurate cost measuring tools available, even in the business sector, so the new design for reimbursement to the provider and facilities was based on a perception of the value of one service relative to another, reflecting some estimate of work and time, ie, a heart surgery was valued higher than a routine physician visit. This relative value unit, even today, remains a part of the equation that sets reimbursement. But it has no linear relationship to cost.

Insurance, by separating the consumer from the price of the purchase of health care services (eg, the third party payment system), left the paying consumer completely unaware of the price of medical services. It was not necessary to measure cost because insurance provided a buffer. From the perspective of the consumer, until premiums rose or out of pocket health care expenditures became an untenable portion of the consumer's budget, there was no pressure to demand price transparency or the measurement of cost. Among the undesirable consequences are almost 50 million uninsured individuals in the US, and nearly two thirds of all personal bankruptcies, and the majority of home foreclosures due to medical expenses. ${ }^{25,26}$

Changes in the reimbursement model were particularly troublesome for providers. In setting prices, insurers supported with actuarial analysis of large claims databases were in a much superior negotiating position relative to providers, many of whom did not even have practice management systems from which to pull data to support their negotiation of prices with the insurer. Thus, providers and facilities were left to play on a field in which boundaries and rules were not established based on cost or quality.

Providers and facilities now need to know accurate measured cost. But they perceive the design and implementation of cost systems as an unaffordable expense. This is especially true when prices are dictated by Medicare and third party payers, whom providers have little or no power to control. The result has been the gradual rise in procedural based medicine $^{20}$ and fragmentation of health care delivery by the rise of subspecialty practices and specialty facilities. ${ }^{19}$

The decision to use a relative value approach to develop the synthetic market prices (reimbursement rates), for Medicare was based upon practical rather than theoretical grounds. The relative value approach did not require that each procedure be subjected to a time consuming analysis to determine the value of the resources consumed in the procedure. Instead, current charges were the major input and each procedure was then subjected to a survey of physicians as to the value of one relative to another. Although subsequent updates incorporated practice expense, malpractice, and physician work, the core of the system is still based on survey of the 
perceived relative value or work level. Practice expense is done by survey of existing, not measured cost. The overall effort to keep these synthetic market prices current has proven both difficult and contentious. ${ }^{27}$ Without a continuous and timely updating of all procedural relative value units and a measured cost input, factors such as significant technology changes, cost of practice changes, and best practice patterns cannot be incorporated easily into the reimbursement scheme.

\section{Previously proposed solutions to procedure costing}

Managerial costing techniques were revolutionized by the creation and adoption of activity based costing for the complex mass and job shop producer ${ }^{28}$ (hospitals are complex job shops). These techniques were facilitated by the development of decision support systems that can capture the resources used to provide a service or procedure. These techniques with greater or lesser degrees of sophistication have been applied in selected health care settings. Numerous publications exist on costing of radiology services, operating room services, and a multitude of provider and facility services. National level proposals that include measuring costs have included the recent time-based activity-based costing proposal by Kaplan and Porter $^{29}$ and the Prometheus Payment System. ${ }^{4}$ Well-known health care facilities have applied these concepts to manage costs and improve efficiency and quality $^{30}$ (http://blogs.hbr.org/2013/10/intelligent-redesignof-health-care/).

Most proposals have two major limitations when moving forward to a national level costing system. First, they require implementation of cost systems on a case-by-case basis. This strategy might work in a system like the Veterans Administration Healthcare facilities where a decision support system exists and facilities, providers, satellites, and home based care are captured and communicated to a central system. However, the US health care system is a cottage industry composed of hospitals of various types, some aligned with

Table 2 Health delivery systems and facilities

\begin{tabular}{ll}
\hline Delivery systems & Organizations/facilities \\
\hline - Non-integrated delivery systems & - Academic medical centers \\
- Integrated delivery systems & - Community hospitals \\
- Accountable care organizations & - Safety net hospitals \\
- Patient-centered medical homes & - Specialty hospitals \\
- Free-standing private \\
clinics/offices \\
- Community health centers \\
- Extended care/skilled \\
nursing facilities \\
\hline
\end{tabular}

academic medical centers, private practices, free standing ambulatory service centers, and other entities included in Table 2. Without a coherent plan, implementing a general cost management system across such a broad landscape is inconceivable. Large facilities may find the return on investment of purchasing and implementing a cost management system acceptable, but for smaller practices, the costs would likely be prohibitive. Further, standardized cost methodologies for health care services have not been developed or tested for accuracy.

\section{Our proposal}

Our solution, described in detail, follows. The determination of rcosts should follow the procedures developed in the commercial sector for products and services when using an activity-based costing approach. Where possible a causal relationship between the cost incurred and the procedures performed should be identified. These costs should be assigned to the procedures using cost drivers that capture the identified causal relationship; for example, machine hours should be used to assign costs such as machine maintenance that vary with the number of machine hours dedicated to the procedure. As another examble, admission costs should be assigned to each patient admitted. There are always some costs (the cost of space is often one of them) that cannot be causally assigned to procedures. These costs can be assigned to the procedures using general purpose, non-causal bases such as head count or number of procedures performed.

We propose a standard health care costing system that allows for automated costing of procedures and services that can be updated regularly. Health care providers would submit their costs in accordance with the standard health care cost system design. The unique feature of our proposal is that only a limited number of representative medical facilities would be needed to provide the source data, as discussed further.

This process would develop new reimbursement rates that are based upon synthetic market prices that equal the rcosts of each procedure. (Actual and accurate costs are terms to be avoided since the actual cost of a procedure cannot be measured). The term rcosts of a procedure and procedural costs would be used interchangeably to represent the cost of a procedure that is reported by a cost system plus an imputed profit margin adjusted for complexity and risk factors and the geographic location of the facility.

Under our approach reimbursement rates are determined using the formula: 
Cost-based reimbursement rate $=$

Reported procedure cost * Specialty profit margin

* Complexity factor* Geographic factor.

The advantage of this approach is that while procedural rcosts can be determined on a regular basis and used to update the synthetic market prices at relatively low cost, the imputed profit margins, which are expensive to estimate, can be updated on a less frequent basis. This alternative approach therefore splits the development of reimbursement rates into two separate steps; one low cost, namely determining procedure rcosts, and the other high cost, namely imputing profits.

The reimbursement formula previously shown can be modified to take into account a wide range of factors that differentially affect the cost of a procedure. ${ }^{31}$ Typically, to avoid excess complexity only factors that make a significant difference in rcosts are incorporated into the calculations.

Determining good estimates of procedure rcosts does not require developing cost systems at every medical facility in the country. Such an approach would be nearly impossible, not to mention unacceptable, since the number of experienced cost system designers available is too small to enable the thousands of systems to be implemented in a reasonable time frame and the high cost of each implementation renders the entire approach too expensive. Porter and Lee $^{32}$ argue that integrated cost units make measurement of rcosts easier. But this requires that integrated practice units (IPUs) be put in place for every disease. Once in place, they can be used as an alternative approach, but this will take a long time. Our solution is faster and considerably less expensive.

The medical facilities chosen to generate the cost information should be broadly representative of systems across the country. Representative types of delivery systems and facilities are listed in Table 2. Pairs representing selections from each category would be chosen, realizing that some pairs are neither logical, feasible nor necessary (eg, community health center and integrated delivery systems). The cost systems that are implemented in these facilities should be activity-based and carefully designed to report "accurate" procedure rcosts. Examples of three health care systems that have done so are given in a recent Harvard Business Review article by Kaplan and Porter. ${ }^{29}$ The heterogeneity of the examples they cite precludes (nor was it intended to provide $)^{24}$ generalizable conclusions, but rather illustrates the concept. While Kaplan and Porter provide a general summary of the seven steps required for cost measurement, the inertia for each system to complete those steps is substantial, and hence somewhat unrealistic. Our approach circumvents that limitation.

The cost systems at the chosen facilities only have to be run once a year to provide annual rcosts for each procedure. These annual rcosts can be used to update the reimbursement rates. If the selected facilities focus on increasing the cost efficiency with which they perform each procedure, then a steady pressure will be built into the reimbursement rates for all medical facilities to become more efficient. Procedures should become less expensive over time. The outcome of using rcosts to set reimbursement rates should be a steady downward pressure on health care costs.

The imputed profit margins used for each medical specialty could be derived using relative value analyses. This approach has the advantage that it also incorporates both complexity and risk adjustments into the new reimbursement rates. Since the underlying approach for these analyses has been developed for the existing reimbursement approach, imputed profit margins can be derived for the limited number of medical specialties that exist. As mentioned previously, a major limitation of the current relative value approach is the amount of effort it takes to derive the tens of thousands of market prices required at the procedure level. Establishing complexity factors for each procedure is time consuming, but the existing relative values can be used to derive the initial profit margins and then a regular updating procedure like the current Relative Value Scale Update Committee system could be developed and implemented. The much smaller number of specialties (about 130 - http://www.abms.org/ who we help/physicians/specialties.aspx) allows the more complex but effective relative value approach to be used where it is most needed - setting profit margins. The simpler and less expensive determination of procedure rcosts can be applied at the procedure level.

One simple solution to the challenge of determining imputed profit margins is to assign each specialty a single margin based upon relative value principles applied at the specialty level. The advantage of a single profit margin per specialty is the significantly reduced number of profit margins that have to be determined compared to the current relative value approach. The disadvantage of this approach in a pay per procedure world is that it creates incentives to perform the lowest relative value procedures and disincentives to perform the highest relative procedures. These incentives are created because the constant margin assumption does not reward the extra skill required of the high relative value procedures. 
As payment reform moves the country toward partial or full capitation, a single profit margin per specialty no longer creates these inappropriate incentives as long as the mix of procedures performed by a facility is relatively stable. In the short-term therefore, a possible solution to avoid creating inappropriate incentives is to use the existing relative value rates for facilities that are under pay per procedure reimbursement and switch to single profit margin rates as they shift to partial or full capitation reimbursement.

If the mix of procedures performed is not stable but for example depends upon the patient group for whom the contract is being negotiated, then a more sophisticated approach to the determination of specialty profit margins is required. The most sophisticated approach is to impute a unique profit margin for each procedure based upon relative value principles applied at the procedure level. The advantage of this approach is that the incentives to perform different procedures would accurately reflect their relative values thus allowing the approach to be used under both approaches to reimbursement. The disadvantage is the complexity, which recapitulates that of the relative value one it seeks to replace.

A practical compromise that could be adopted is to create a number of relative value ranges for procedures and to use these to adjust the imputed profit margins. For example, suppose that a complexity range with five levels is adopted. The lowest range might have a relative value of one and the highest a relative value of two. The imputed profit margins for procedures that fall into the highest range would be twice that of procedures that fall into the lowest range. This relative levels approach illustrates an important point that is often overlooked - the determination of reimbursement rates does not have to be exact - just close enough. It is not feasible to determine procedure rcosts to one figure right of the decimal, nor are the relative values of the current approach anywhere near that accurate. Artificially imposing apparent accuracy on an imprecise process simply wastes effort and sends inaccurate messages.

Geographic variations (rural versus urban, high versus low cost states etc) can be incorporated into the approach by multiplying the geographic neutral reimbursement rates for each procedure by an appropriate geographic factor. For expedience the geographic factors currently in use could be adopted but a better solution would be to use the rcosts structure of the representative facilities and that of carefully chosen facilities in various locations to create more accurate geographic factors.

None of the calculations described are complex in theory; it is simply the number of them that renders any approach, on the surface, overwhelming. Fortunately, each of these steps can be undertaken in parallel. The cost systems can be implemented at the designated locations independently of all the other steps. So can the assignment of complexity levels to each procedure. Simultaneously, the imputed profit margins for each specialty can be developed. At the same time, the first generation regional adjustment factors can be reviewed or determined. Subsequently, they can be based upon more sophisticated approaches that rely upon the rcosts. If the overall impact of the change has to be reimbursement neutral (ie, the total reimbursement in the year of adoption is the same), then neutrality adjustments to each rate can be made as the very last step in the process. The most expedient approach is to use a single cost neutrality factor to modify all of the prices by the same ratio. This ability to perform each step in parallel is not shared by the relative value approach where all except the regional adjustments are incorporated into the single step of setting relative values. The advantage of parallelism is that updating can be undertaken at different frequencies. For example, rcosts can be updated yearly, complexity levels every 5 years (with one fifth of all procedures updated every year), imputed profit margins per specialty every 2 years (with one half updated every year), and regional adjustment factors every 5 years (all at once). Under this type of program reimbursement rates are kept reasonably current with at most a 5 year lag. If this lag is deemed too long, then a different updating schedule can be adopted. In contrast, if annual updating is considered too frequent, then a longer refresh cycle for the rcosts can be implemented.

\section{A cloud-based open access model should be used}

We propose a cloud-based, open access model because it encourages innovation, competition and, where appropriate, business to business cooperation or competition. Publically sharing the structure of these cost systems, the quality of care programs that are in place, and the efficiency programs and their savings, will help the other facilities become more efficient by decreasing the cost and complexity of implementing their own cost, quality, and efficiency systems. A cloud-based, open access model provides an opportunity for accelerated pace of change, user generated consent, and introducing market competition into the process. After the initial cost system implementation, the open access design will invite an accelerated pace of change as experts in costing compete to develop business models using cost and quality to invite change. If the initial rcosts were used to replace 
open-ended subsidies to beneficiaries and price-controlled reimbursements to providers with fixed dollar subsidies instead, the result would effectively shift Medicare from a defined-benefit to a defined contribution approach. Thus the business model would shift from one that is driven by the volume and intensity of services to one that rewards cost effective and efficient care.

\section{Further evidence that it can be done}

Cost systems based upon well-established designs adapted from the commercial sector are used frequently in health care. Using the MeSH terms "health care costs" OR ("health" [All Fields] AND "care" [All Fields] AND "costs" [All Fields]) OR health care costs [All Fields] OR ("healthcare" [All Fields] AND "cost" [All Fields]) OR "healthcare cost" [All Fields], 200 English language articles were identified in a computerized search of the MEDLINE data base completed on June 28th, 2012. Articles that were based on a cost economic analysis were eliminated because they adopt a different and non-applicable definition of cost. Health Care Cost and Utilization Project and other articles that contained the word "cost" but did not include a cost analysis were also eliminated, leaving 106 remaining articles. Of these, 30\% used commercially originated costing methods including activity based costing; $60 \%$ were completed outside of the US, all in countries with national health care systems. Thus, the critical challenge is not implementing cost systems in health care, but to find a practical way to determine system-wide procedure rcosts in the US that can be used to establish reimbursement rates. Our proposal does exactly that.

\section{Implementation requirements}

Successful implementation of this plan depends on a series of factors. Minimal requirements include:

- all sites must use the same activity-based methodology for defining procedures and determining rcosts;

- information is collated, stored, and exchanged using fully interoperable information technology;

- the risk adjustment methodology is identical across sites;

- factor prices and wages are adjusted to reflect geographic variations;

- costs associated with education (students, residents, fellows) are categorized separately;

- data is made publically available, with de-identification of site of care as required;

- the undertaking is cost neutral, at worst, for all sites.
The most logical entity to undertake this initiative is Medicare, as a pilot project under the auspices of the Patient Protection and Affordable Care Act, through the Centers for Medicare and Medicaid Innovation. Applicants wishing to participate would submit applications in response to an RFA Request for application.

\section{Implications for policy makers}

Our proposed approach has broad policy implications beyond the fundamental value of aligning costs and procedures. At the heart of these implications is the shift from the high cost of determining reimbursement rates under the relative value approach to the lower costs associated with the limited application cost-based approach discussed in this paper. We describe some of the more substantive implications below.

By evolving away from the current system, in which financial incentives are buried in the reimbursement rates, academic medical centers, a dominant provider of charity care, will have more protection against large losses when high profit specialties exit. Thus, the subsidy of the uninsured will be visible as profit margins are currently boosted to pay for the uninsured. As the Affordable Care Act reduces disproportionate share payments concomitant with an increase in the insured population, our methodology will provide more clarity on the hidden costs of charity care.

The costing and imputed profit margin can be used to alter the attractiveness and scope of different specialties; for example, increasing the profit margin of a specialty allows the relative attractiveness of that specialty to be increased. Thus, if too few graduating medical students are entering a specialty, its overall imputed profitability can be increased to make that specialty more attractive and thus avoid future shortages in trained practitioners. In contrast, if a specialty is attracting too many students, its overall profitability can be reduced, making it less attractive.

If multiple specialties perform the same procedure, either separate synthetic prices can be developed for each specialty or another round of averaging can be performed to create a single synthetic price for the procedure. This single price approach will create economic pressures for the specialties with the lowest imputed profit levels to perform these procedures, as the average rcost will cause them to look especially profitable. Updating the average will cause the synthetic price to drop as the specialties with the lowest imputed profit levels will come to dominate the provision of certain procedures. Having different prices for each specialty avoids this incentive, but adds complexity to the reimbursement process and 
does not create as great a pressure to reduce reimbursement rates.

Our method facilitates improvements in quality and efficiency. The difference between paid claims values and measured cost can be calculated and the differences between facilities and delivery systems analyzed. Chosen facilities can act as test beds for new approaches. Facilities demonstrating improvements in cost with efficiency and quality measures already standardized by the Association for Healthcare Research Quality can become new leaders for the industry by publishing their results. Evidence-based clinical practice guidelines can be attached to a cost and waste of resources will become visible and be incorporated into the health care delivery models provided at the chosen facilities. Published results allow every facility to choose to stop waste when documented evidence dictates the need for change. Finally, data comparisons and reliability tests can be applied to look at reasons for variations. Measured costing data can be used to complement risk-adjusted models of care, using claims data or charges such as Health Partners Total Cost of Care ${ }^{33}$ and similar models such as Ambulatory Care Groupings published from Johns Hopkins. ${ }^{34}$ Although efficiency is not built into the initial cost system design, the cloud-based business-to-business competition creates a platform to reduce costs while retaining quality, and to drive future efficiencies.

\section{Potential objections}

Decisions made based on the availability of cost information, in light of misplaced perverse financial incentives, will of course bring enormous fear. The most obvious objection will be cuts in specialty reimbursement that will result. But such cuts are already underway, and more are inevitable, with delivery and payment reform. New delivery models (Accountable Care Organizations and Patient Centered Medical Homes) and new payment models (gain sharing, reference pricing/defined contribution, partial or full capitation) are all driving toward reduced and/or more competitive utilization of specialty care. But this is happening without the data to align costs and quality, and hence to optimize the relative utilization of primary versus specialty care. The most likely scenario is that specialty care will be undervalued. Our proposal allows physicians to approach a level playing field relative to insurers and Medicare by the availability of evidence and knowledge and helps to avoid the conundrum of the "must-have" providers. ${ }^{35}$ Our proposal facilitates appropriate valuation of clinical services across the continuum.
In this proposal, we largely leave aside the issue of hospital price lists (charge masters). If anything the obfuscation that methodology introduces is more problematic than that around the RBCA. The underlying cause, however, is the same. The use of reimbursements rather than costs to set prices and to negotiate payment arrangements with payers creates massive misalignment.

\section{Conclusion}

Our proposal focuses on the fundamental question underlying all current health care issues - how do we achieve better value for the health care dollar? To achieve maximum coverage at acceptable expense with acceptable quality, health care will need to become subject to the same forces that have boosted efficiency and value throughout the economy.

Costs will be measured. The process will be transparent to entities that are charged with developing accurate pricing of procedures and services..$^{20}$ Misaligned incentives will be visible and appropriately structured incentives can be created. Waste can be identified and realigned, and efficiency protocols can be developed.

Of great importance, our approach is not predicated on selecting or implementing any specific payment methodology. It is equally applicable and informative to current proposals for bundled/episode of care payments, shared savings, defined contribution plans, patient-centered medical homes, and fee for service medicine.

Our proposal is a web-based informational gathering tool that allows for universal access to data nowhere else available in health care. It places the consumer as the ultimate guarantor of good services, reasonable prices, and sensible trade-offs between health care spending and spending on other goods and services.

\section{Disclosure}

The authors have no conflicts of interest with the material reported in this manuscript.

\section{References}

1. Antos JR, Pauly MV, Wilensky GR. Bending the cost curve through market-based incentives. N Engl J Med. 2012;367(10):954-958.

2. Emanuel E, Tanden N, Altman S, et al. A systemic approach to containing health care spending. $N$ Engl J Med. 2012;367(10):949-954.

3. The Commonweatlh Fund [homepage on the Internet]. Modern Healthcare Health Care Opinion Leaders Survey: Views on Transparency and Pricing; 2010. Avialable from: http://www.commonwealthfund.org/publications/surveys/2010/oct/views-on-transparency-and-pricing. Accessed August 7, 2014.

4. de Brantes F, Rosenthal MB, Painter M. Building a bridge from fragmentation to accountability - the Prometheus Payment model. $N$ Engl $J$ Med. 2009;361(11):1033-1036. 
5. Robinson JC, MacPherson K. Payers test reference pricing and centers of excellence to steer patients to low-price and high-quality providers. Health Aff (Millwood). 2012;31(9):2028-2036.

6. Kanavos P, Reinhardt U. Reference pricing for drugs: is it compatible with US health care? Health Aff (Millwood). 2003;22(3):16-30.

7. Frakt AB, Mayes R. Beyond capitation: how new payment experiments seek to find the 'sweet spot' in amount of risk providers and payers bear. Health Aff (Millwood). 2012;31(9):1951-1958.

8. Porter ME. What is value in health care? NEngl J Med. 2010;363(26): 2477-2481.

9. Baicker K, Chandra A. Aspirin, angioplasty and proton beam therapy: The economics of smarter health care spending. Federal Reserve Bank of Kansas City. Jackson Hole Economic Policy Symposium, 2011. http:// www.kansascityfed.org/publicat/sympos/2011/Baicker-Chandra_final. pdf.

10. Institute of Medicine [homepage on the Internet]. Best care at lower cost: the path to continuously learning health care in America; 2012. Available from: http://www.iom.edu/Reports/2012/Best-Care-at-LowerCost-The-Path-to-Continuously-Learning-Health-Care-in-America aspx. Accessed August 7, 2014.

11. Cassel CK, Guest JA. Choosing wisely: helping physicians and patients make smart decisions about their care. JAMA. 2012;307(17): 1801-1802.

12. Clinical evidence [homepage on the Internet]. Available from: http:// clinicalevidence.bmj.com. Accessed August 7, 2014.

13. Grady D, Redberg RF. Less is more: how less health care can result in better health. ArchInternMed. 2010;170(9):749-750.

14. Garner S, Littlejohns P. Disinvestment from low value clinical interventions: NICEly done. BMJ. 2011;343:d4519.

15. Prasad V, Vandross A, Toomey C, et al. A decade of reversal: an analysis of 146 contraindicated medical practices. Mayo Clin Proc. 2013;88(8): 790-798

16. Hussey PS, Ridgely MS, Rosenthal MB. The PROMETHEUS bundled payment experiment: slow start shows problems in implementing new payment models. Health Aff (Millwood). 2011;30(11):2116-2124.

17. de Brantes F, Rastogi A, Soerensen CM. Episode of care analysis reveals sources of variations in costs. Am J Manag Care. 2011;17(10): e383-e392.

18. Cooper R, Kramer TR. RBRVS costing: the inaccurate wolf in expensive sheep's clothing. JHealth Care Finance. 2008;34(3):6-18.

19. Cooper R, Kramer TR. Revenue-based cost assignment: a potent but hidden threat to the survival of the multispecialty medical practice. Acad Med. 2010;85(3):538-547.

20. Ginsburg PB. Fee-for-service will remain a feature of major payment reforms, requiring more changes in Medicare physician payment. Health Aff (Millwood).2012;31(9):1977-1983.
21. Ginsburg PB, Berenson RA. Revising Medicare's physician fee schedule - much activity, little change. $N$ Engl J Med. 2007;356(12): 1201-1203.

22. Goodman J. Perspective: What is consumer directed health care? 2006 www.ncpa.org/email/hlthaff.25.w540v1.pdf.

23. Mayes R. The origins, development, and passage of Medicare's revolutionary prospective payment system. J Hist Med Allied Sci. 2007;62(1):21-55.

24. Hsiao WC, Braun P, Dunn D, Becker ER, DeNicola M, Ketcham TR. Results and policy implications of the resource-based relative-value study. N Engl J Med. 1988;319(13):881-888.

25. Robertson CT, Egelhof R, Hoke M. Get sick, get out: the medical causes of home mortgage foreclosures. Health Matrix Clevel. 2008;18(1): 65-104.

26. Himmelstein DU, Thorne D, Warren E, Woolhandler S. Medical bankruptcy in the United States, 2007: results of a national study. Am J Med. 2009;122(8):741-746.

27. Laugesen MJ, Wada R, Chen EM. In setting doctors' Medicare fees, CMS almost always accepts the relative value update panel's advice on work values. Health Aff (Millwood). 2012;31(5):965-972.

28. Cooper R, Kaplan RS. Measure costs right: Make the right decisions. Harv. Bus Rev. 1988;66(5):96-103.

29. Kaplan RS, Porter ME. How to solve the cost crisis in health care. Harv Bus Rev. 2011;89(9):46-52.

30. Blackmore CC, Mecklenburg RS, Kaplan GS. At Virginia Mason, collaboration among providers, employers, and health plans to transform care cut costs and improved quality. Health Aff (Millwood). 2011;30(9): 1680-1687.

31. Brekke KR, Levaggi R, Siciliani L, Straume OR. Patient mobility, health care quality and welfare. J Econ Behavior and Organization. 2014;105:140-157.

32. Porter M, Lee TH. The strategy that will fix health care. Harv. Bus. Rev. 2013;91(10):50-70.

33. Lindquist, Tammie J. Plymouth M, Power, Robert P. St. Paul M, Kitching, et al., Inventors; Group Health Plan, Inc. Bloomington, $\mathrm{MN}$, assignee. Method and computer program product for measuring and utilizing efficiency of medical resource and services providers. Patent US 20100114611 AI. Publication Date. May 6, 2010.

34. Weiner JP, Starfield BH, Lieberman RN. Johns Hopkins Ambulatory Care Groups (ACGs). A case-mix system for UR, QA and capitation adjustment. HMO Pract. 1992;6(1):13-19.

35. Berenson RA, Ginsburg PB, Christianson JB, Yee T. The growing power of some providers to win steep payment increases from insurers suggests policy remedies may be needed. Health Aff (Millwood). 2012;31(5):973-981.
Innovation and Entrepreneurship in Health

\section{Publish your work in this journal}

Innovation and Entrepreneurship in Health is an international, peer reviewed, open access journal publishing original research, reports, reviews and commentaries on innovation and entrepreneurship in health. Special focus will be given to the theory, process, and practice of innovation and entrepreneurship by individuals and organizations

\section{Dovepress}

within the health care context globally. The manuscript management system is completely online and includes a very quick and fair peer review system, which is all easy to use. Visit http://www.dovepress.com/ testimonials.php to read real quotes from published authors. 